\title{
A Correlation-Based Electromagnetic Time Reversal Technique to Locate Indoor Transient Radiation Sources
}

\author{
Qi Li, Zhao-yang Wang, Yan-zhao Xie, Senior Member, IEEE, Farhad Rachidi, Fellow, IEEE and \\ Marcos Rubinstein, Fellow, IEEE
}

\section{NOMENCLATURE}

\begin{abstract}
To overcome the multipath interference in locating transient electromagnetic (EM) radiation sources in an indoor environment, we propose a criterion that calculates the correlation between back-propagated signals from observation points, to be used in Electromagnetic Time Reversal (EMTR) algorithms. The method introduced in this paper has three main advantages with respect to classical methods that use full-wave simulations and other criteria, such as maximum field strength. First, compared with full-wave techniques in the back-propagation phase, the proposed correlation-based method utilizes approximated transfer functions from the Ray Tracing technique, which can improve the computation efficiency. Second, an inverted-loss model is used for the back-propagation, which could reduce the localization error caused by multipath effects due to signal attenuation and time delay. Third, the proposed correlation criterion has weak correlation with the source characteristics, which makes it applicable to the localization in indoor reflective environments with only two observation points. Several numerical simulations are carried out to assess the performance of the proposed method. The results indicate that the proposed correlation-based EMTR technique is able to locate radiation sources accurately and efficiently in indoor reflective environments.
\end{abstract}

Index Terms-transient electromagnetic radiation sources; indoor environment; Electromagnetic Time Reversal; correlation; Ray Tracing technique.
Variables:

$\begin{array}{cc}E & \text { Electric field } \\ E_{T R} & \text { Time-reversed electric field } \\ G & \text { Transfer function } \\ \text { Cov } & \text { Covariance } \\ \text { Var } & \text { Variance } \\ h & \text { Impulse response } \\ \Gamma & \text { Reflection coefficient } \\ K, \kappa & \text { Proportion coefficient related to impulse } \\ \mu & \text { response } \\ \Psi & \text { Mean value }\end{array}$

Parameters:

$\begin{array}{cc}r_{s} & \text { Position of source } \\ r_{x} & \text { Position of observation point } x, x \text { being a positive } \\ & \text { integer } \\ r_{o} & \text { Position of guessed source } \\ f & \text { Frequency } \\ S & \text { Source signal } \\ \theta & \text { Reflection angle } \\ n & \text { Reflection order } \\ k & \text { Wave number }\end{array}$

$d \quad$ Distance between source and receiver

$d_{n} \quad$ Distance between receiver and $n$-th image of source $l, l_{0}, l_{1}$, $l_{2}, l_{r}, l_{t}$ Engineering, Beijing 100854, China (e-mail: liqil@stu.xjtu.edu.cn)

Y.-Z. Xie is with the State Key Laboratory of Electrical Insulation and Power Equipment, School of Electrical Engineering, Xi'an Jiaotong University, Xi'an 710049, China (e-mail: yzxie@xjtu.edu.cn)

Z.-Y. Wang and F. Rachidi are with the Swiss Federal Institute of Technology, Lausanne 1015, Switzerland (e-mail: zhaoyang.wang@epfl.ch; farhad.rachidi@epfl.ch).

M. Rubinstein is with the University of Applied Sciences of Western Switzerland, Yvderon-les-bains 1400, Switzerland (e-mail: rubinstein.m@gmail.com).
(C) 2019 IEEE. Personal use of this material is permitted. Permission from IEEE must be obtained for all other uses, in any current or future media, including reprinting/republishing this material for advertising or promotional purposes, creating new collective works, for resale or redistribution to servers or lists, or reuse of any copyrighted component of this work in other works. 


\section{INTRODUCTION}

$\mathrm{T}$ he localization of EM radiation sources in a medium involving multipath propagation is a challenging task, essentially because of the interference at the receivers caused by the path-overlap phenomenon [1-2]. In certain applications, such as the indoor localization based on UWB (ultra wide-band) systems [3-5], EM waves traveling between nodes experience multiple reflections which affect the received signals and decrease the location accuracy. Due to reflections, the received signal contains not only the line-of-sight (LOS) wave but also the reflected components (Non-line-of-sight, NLOS) [6], resulting in multiple 'ghost' sources. Table I presents a qualitative comparison of the conventional passive localization methods used in indoor environments [7]. As Table I shows, the accuracy of the first four methods requires the existence of the LOS wave. When the LOS wave is disturbed by the multipath effects, the accuracy of these methods is significantly reduced. Moreover, the number of observation points required by these methods is more than three in a 2-D location. The accuracy of the fingerprint method depends on the source characteristics, which are unknown in most application scenarios [8]. The proximity method usually makes use of a large number of observation points [9]. The presented comparison in Table I highlights the need of enhanced localization techniques in multipath environments, whose performance is independent of the source characteristics and requires a minimal number of measuring points.

TABLE I

PERFORMANCE COMPARISON OF CONVENTIONAL INDOOR LOCALIZATION METHODS

\begin{tabular}{|l|c|c|c|c|}
\hline \multicolumn{1}{|c|}{ Method } & $\begin{array}{c}\text { Time } \\
\text { Synchroni- } \\
\text { zation } \\
\text { Requireme- } \\
\text { nt }\end{array}$ & $\begin{array}{c}\text { Can be used } \\
\text { in NLOS } \\
\text { Environme- } \\
\text { nt }\end{array}$ & $\begin{array}{c}\text { Related to } \\
\text { Source } \\
\text { Characteris } \\
\text {-tic }\end{array}$ & $\begin{array}{c}\text { Minimum } \\
\text { Number of } \\
\text { Measuring } \\
\text { Points (2-D } \\
\text { Localizatio- } \\
\text { n) }\end{array}$ \\
\hline $\begin{array}{l}\text { Received } \\
\text { Signal } \\
\text { Strength [7] }\end{array}$ & Low & No & No & 3 \\
\hline $\begin{array}{l}\text { Time of } \\
\text { Arrival [7] }\end{array}$ & High & No & No & 3 \\
\hline $\begin{array}{l}\text { Time } \\
\text { Difference } \\
\text { of Arrival } \\
\text { [7] }\end{array}$ & High & No & No & 3 \\
\hline $\begin{array}{l}\text { Angle of } \\
\text { Arrival [7] }\end{array}$ & High & No & No & 4 \\
\hline $\begin{array}{l}\text { Fingerprint } \\
\text { [8] }\end{array}$ & Low & Yes & Yes & $\begin{array}{c}\text { Database } \\
\text { required }\end{array}$ \\
\hline $\begin{array}{l}\text { Proximity } \\
\text { [9] }\end{array}$ & Low & Yes & No & $2 \sim \infty$ \\
\hline
\end{tabular}

Some mitigation techniques for multipath interference have been proposed for many relatively simple applications [10-11]. But inaccuracies are still observed when these techniques are used in some complex scenarios such as in environments without LOS wave [12-13]. By modeling propagation through walls and specular reflections in an enclosed room, Setlur et al. [14] derived a multipath model, which could effectively reduce the number of false positives in the original beamformed image. With the help of a known plan, Meissner et al. [15-16] achieved accurate and robust anchor localization in an indoor environment by using reflected signal paths that have been mapped to virtual anchors.

However, the localization accuracy of these methods is dependent upon the availability of a detailed EM propagation model and the advanced knowledge of the features of the source. For example, in order to locate mobile terminals using a single base station, Porretta et al. [17-18] proposed a novel algorithm that can perform well when operating in a microcellular environment with perfect channel-parameter estimation. Abbasi et al. [19] introduced an improved procedure in multipath environments by considering skewness as new statistical information, and they were able to effectively reduce the localization error. There is no doubt that a good EM propagation model representing accurately the reality helps to improve the localization accuracy. However, the computational efficiency would clearly decrease in turn [20]. In addition, the source signal is unknown in most cases, which would increase the difficulty of localization.

Recently, the EMTR technique has attracted considerable attention in the field of active source and target localization [21-23]. It has been found that making use of the time-reversal (TR) invariance of Maxwell's equations, the time reversed electromagnetic waves would undergo, in reverse, the same reflection, refraction and scattering processes as in the forward propagation, and refocus back to the original position of the radiation source. Different studies have shown the potential of EMTR to realize the focusing of waves inside metallic cavities such as reverberation environments by using the infinite number of reflections caused by highly reflective materials [24-25]. However, the effectiveness of the method to locate radiation sources in an indoor reflective environment (composed of ground, walls, etc.) has not been demonstrated, especially when only two observation points are available.

In this paper, an EMTR technique based on the magnitude and phase correlation criteria is proposed to locate radiation sources in a reflective medium. The rest of the paper is organized as follows. Section II introduces the proposed method which is derived in the frequency domain. Section III establishes an equivalent propagation model, and the required transfer functions are approximated by combining geometric modeling and the Ray Tracing technique, in order to accelerate computation in the back-propagation step. In Section IV, the approach is tested with numerical simulations and the effects of influencing factors related to the spatial resolution are investigated. In Section V, the approach is validated in a full wave propagation environment implemented within CST Microwave Studio. Finally, conclusions are given in Section VI.

\section{CORRELATION-BASED CRITERION EMTR APPLIED TO SOURCE LOCALIZATION}

\section{A. Basic Principle}

In this section, an EMTR technique for source localization using a correlation-based criterion is described in four steps, namely (i) measuring of the direct-time radiation, (ii) 
time-reversing the measured signals, (iii) back-injecting them into the medium by numerical simulations, and (iv) estimating the source position using the proposed correlation criterion.

1) Radiation

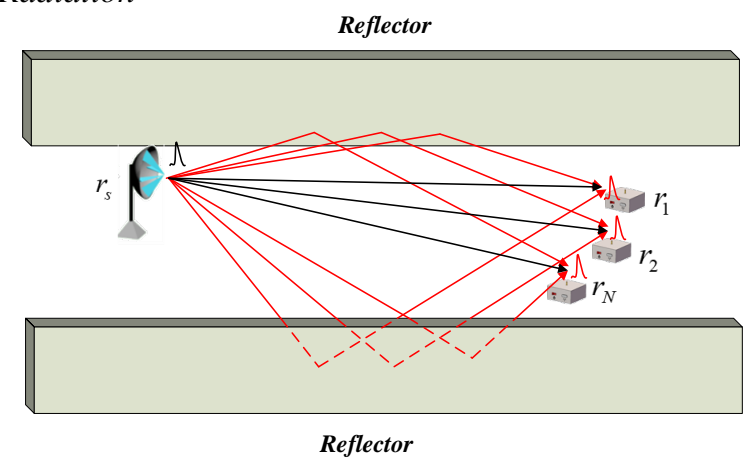

Fig. 1. Propagation model in a multipath environment.

Let us consider a transient radiation source located at a position $r_{s}$ in an indoor environment, as shown in Fig. 1. An array consisting of $X$ sensors is set up in the far field region, at positions $r_{x}(x=1,2,3, \ldots, X)$. In order to reduce the complexity in the derivation process caused by the convolution operation in the time domain, the method will be developed in the frequency domain. Therefore, the radiated electric field (E-field) at the observation point $r_{x}$ can be expressed as

$$
E\left(r_{s}, r_{x}, f\right)=S(f) \cdot G\left(r_{s}, r_{x}, f\right)
$$

where $S(f)$ is the radiated signal with the frequency $f$ and $G\left(r_{s}, r_{x}, f\right)$ represents the transfer function between the source position and the considered observation point, which is affected by the multipath environment including losses, dispersion and time delay [26-27].

2) Time Reversal

According to the EMTR method, the electric field at the observation point (given by (1)), is time reversed before being back-propagated. The time reversal corresponds to the complex conjugate operation in the frequency domain [28]. Thus, the time-reversed signal is given by

$$
E^{*}\left(r_{s}, r_{x}, f\right)=S^{*}(f) \cdot G^{*}\left(r_{s}, r_{x}, f\right)
$$

\section{3) Back Propagation}

A characteristic of the classical time reversal technique is that, although the phase of the time-reversed, back-propagated signal is the same as that of the input signal at the source position, its magnitude is modified by the square of the transfer function between the source and the sensor [29]. Usually, for a complex environment, the transfer function is characterized by a large number of peaks and nulls [30]. Thus, classical criteria such as the maximum signal energy or the maximum peak signal might fail to accurately locate the source [31].

An inverted-loss back-propagation model was proposed in [32], which makes use of an inverted filter to equalize the propagation effects. Following the strict rule of time-reversal invariance, the model has been verified to compensate for the ground losses in lightning strike localization [32]. Similar to this inverted-loss model, in order to overcome the multipath effect including time delay, dispersion and amplitude attenuation, the time-reversed copy of the transfer function $G^{*}\left(r_{x}, r_{o}, f\right)$ between the observation point $r_{x}$ and a given guessed source position $r_{o}$ is calculated by building the propagation model, and then the inverted-loss model is used in the back-propagation phase. Thus, the back-propagated field at $r_{o}$ is expressed as

$$
E_{\text {TR }}\left(r_{x}, r_{o}, f\right)=\frac{E^{*}\left(r_{s}, r_{x}, f\right)}{G^{*}\left(r_{x}, r_{o}, f\right)}=S^{*}(f) \cdot \frac{G^{*}\left(r_{s}, r_{x}, f\right)}{G^{*}\left(r_{x}, r_{o}, f\right)}
$$

Alternatively, one can also follow Equation (4) below, which means that the time-reversed signal $E^{*}\left(r_{s}, r_{x}, f\right)$ is re-radiated to obtain the back-propagated field and then divided by $\left|G\left(r_{x}, r_{o}, f\right)\right|^{2}$ to compensate for the propagation effects.

$$
E_{T R}\left(r_{x}, r_{o}, f\right)=\frac{S^{*}(f) \cdot G^{*}\left(r_{s}, r_{x}, f\right) \cdot G\left(r_{x}, r_{o}, f\right)}{\left|G\left(r_{x}, r_{o}, f\right)\right|^{2}}
$$

\section{4) Correlation Criterion}

From Equation (3), the ratio $\frac{G^{*}\left(r_{s}, r_{x}, f\right)}{G^{*}\left(r_{x}, r_{o}, f\right)}$ will become equal to 1 if $r_{o}=r_{s}$. In this case, (3) reduces to

$$
E_{T R}\left(r_{x}, r_{o}, f\right)=S^{*}(f)
$$

Equation (5) implies that the signals back-propagated by each transmitter will have similar characteristics to the incident waveform at the source position $r_{s}$. If there are two transmitters at $r_{1}$ and $r_{2}$, the back-propagated signals at the source position $r_{s}$ would be the same, namely

$$
E_{T R}\left(r_{1}, r_{s}, f\right)=E_{T R}\left(r_{2}, r_{s}, f\right)=S^{*}(f)
$$

Note that, in practice, some difference will exist due, for instance, to numerical effects and approximations in the propagation model.

In other words, by comparing the similarity of back-propagated signals from each transmitter in the target area, we can identify the position of the source.

In order to quantify the similarity of back-propagated signals, we use the Pearson correlation coefficient, which has been verified to be an efficient tool to describe the linear correlative degree of two variables [33]. Its value ranges from -1 to +1 . A correlation coefficient equal to 1 corresponds to a perfect positive correlation, while a value equal to -1 corresponds to a perfect negative correlation. In other words, the greater the absolute value of the correlation coefficient, the stronger the correlation and vice versa [34].

Based on the analysis above, the back-propagated signals are almost same at the source position, which means that the value of the correlation coefficient calculated at that source position would be close to +1 . In order to identify the source position, the values of the correlation coefficients are obtained, and then the point with the maximum value is identified as the source position. Therefore, the estimated source position is the position satisfying the following equation. 


$$
\begin{aligned}
r_{\text {estimated }} & =\left.\arg \right|_{r_{o}} \max \left(\rho_{E_{T R}\left(r_{1}, r_{o}, f\right) E_{T R}\left(r_{2}, r_{o}, f\right)}\right) \\
& =\left.\arg \right|_{r_{o}} \max \left(\frac{\operatorname{cov}\left(E_{T R}\left(r_{1}, r_{o}, f\right), E_{T R}\left(r_{2}, r_{o}, f\right)\right)}{\sqrt{\operatorname{Var}\left(E_{T R}\left(r_{1}, r_{o}, f\right)\right) \cdot \operatorname{Var}\left(E_{T R}\left(r_{2}, r_{o}, f\right)\right)}}\right)
\end{aligned}
$$

where $\operatorname{cov}\left(E_{T R}\left(r_{1}, r_{o}, f\right), E_{T R}\left(r_{2}, r_{o}, f\right)\right)$ represents the covariance between $E_{T R}\left(r_{1}, r_{o}, f\right)$ and $E_{T R}\left(r_{2}, r_{o}, f\right)$. $\operatorname{Var}\left(E_{T R}\left(r_{1}, r_{o}, f\right)\right)$ and $\operatorname{Var}\left(E_{T R}\left(r_{2}, r_{o}, f\right)\right)$ are the variances of $E_{T R}\left(r_{1}, r_{o}, f\right)$ and $E_{T R}\left(r_{2}, r_{o}, f\right)$, respectively [34].

Strictly speaking, the magnitude and the phase, which indicate the information of the signal, are related to the frequency, but independent of each other. From Equation (7), because the method is derived totally in the frequency domain, two types of correlation criteria based on either the magnitude or the phase of the back-propagated signals can be obtained, by calculating the coefficient of the magnitude in Equation (8) or the phase in Equation (9) of the back-propagated signals, respectively.

$$
\begin{aligned}
& r_{\text {estimated }}=\left.\arg \right|_{r_{o}} \max \left(\frac{\operatorname{cov}\left(E_{T R 1}(f), E_{T R 2}(f)\right)}{\sqrt{\operatorname{Var}\left(E_{T R 1}(f)\right) \cdot \operatorname{Var}\left(E_{T R 2}(f)\right)}}\right) \\
& r_{\text {estimated }}=\left.\arg \right|_{r_{o}} \max \left(\frac{\operatorname{cov}\left(\varphi_{T R 1}(f), \varphi_{T R 2}(f)\right)}{\sqrt{\operatorname{Var}\left(\varphi_{T R 1}(f)\right) \cdot \operatorname{Var}\left(\varphi_{T R 2}(f)\right)}}\right)
\end{aligned}
$$

where $E_{T R 1}(f), E_{T R 2}(f)$ and $\varphi_{T R 1}(f), \varphi_{T R 2}(f)$ are the magnitude and the phase of $E_{T R}\left(r_{1}, r_{o}, f\right)$ and $E_{T R}\left(r_{2}, r_{o}, f\right)$, respectively.

Based on the above considerations, the proposed method for locating a transient radiation source can be summarized in the following steps:

1) Record the radiation field signal $E\left(r_{s}, r_{x}, t\right)$ generated by the transient radiation source at observation points $r_{x}(x=1,2,3, \ldots) \cdot r_{s}$ represents the source position.

2) Time-reverse the signal, $E\left(r_{s}, r_{x}, T-t\right)$, and Fourier transform to obtain $E^{*}\left(r_{s}, r_{x}, f\right)$.

3) Build the propagation environment model and obtain the transfer function $G\left(r_{x}, r_{o}, f\right)$ between each observation point and all guessed positions $r_{o}(o=1,2,3, \ldots)$ in the target area. Then calculate the conjugate functions $G^{*}\left(r_{x}, r_{o}, f\right)$.

4) Choose any two time reversal signals $E^{*}\left(r_{s}, r_{1}, f\right), E^{*}\left(r_{s}, r_{2}, f\right)$ at observation points $r_{1}, r_{2}$ and calculate the back-propagated signals $E_{T R}\left(r_{1}, r_{o}, f\right)$, $E_{T R}\left(r_{2}, r_{o}, f\right)$ at guessed position $r_{o}$ as follows

$$
\begin{aligned}
& E_{T R}\left(r_{1}, r_{o}, f\right)=E^{*}\left(r_{s}, r_{1}, f\right) / G^{*}\left(r_{1}, r_{o}, f\right)=E_{T R 1}(f) e^{j \varphi_{T R 1}(f)}(10) \\
& E_{T R}\left(r_{2}, r_{o}, f\right)=E^{*}\left(r_{s}, r_{2}, f\right) / G^{*}\left(r_{2}, r_{o}, f\right)=E_{T R 2}(f) e^{j \varphi_{T R 2}(f)}(11)
\end{aligned}
$$

where $E_{T R 1}(f), E_{T R 2}(f)$ and $\varphi_{T R 1}(f), \varphi_{T R 2}(f)$ are the magnitude and the phase of $E_{T R}\left(r_{1}, r_{o}, f\right)$ and $E_{T R}\left(r_{2}, r_{o}, f\right)$, respectively.

5) Calculate the value of the Pearson correlation coefficient using the magnitudes $E_{T R 1}(f), E_{T R 2}(f)$ or the phases $\varphi_{T R 1}(f)$, $\varphi_{T R 2}(f)$ at the guessed points $r_{o}$ and pick up the point with the maximum value of the correlation coefficient, which corresponds to the source position.

$$
\begin{aligned}
& r_{\text {estimated }}=\left.\arg \right|_{r_{o}} \max \left(\frac{\operatorname{cov}\left(E_{T R 1}(f), E_{T R 2}(f)\right)}{\sqrt{\operatorname{Var}\left(E_{T R 1}(f)\right) \cdot \operatorname{Var}\left(E_{T R 2}(f)\right)}}\right) \\
& r_{\text {estimated }}=\left.\arg \right|_{r_{o}} \max \left(\frac{\operatorname{cov}\left(\varphi_{T R 1}(f), \varphi_{T R 2}(f)\right)}{\sqrt{\operatorname{Var}\left(\varphi_{T R 1}(f)\right) \cdot \operatorname{Var}\left(\varphi_{T R 2}(f)\right)}}\right)
\end{aligned}
$$

\section{B. Numerical Simulation}

In order to assess the performance of the proposed method, a simple radiation model is considered within CST Microwave Studio (CST-MWS), which uses the finite integration technique in the time domain. Regarding the transient radiation source, a dipole is applied as the radiation antenna, which is placed at $(0 \mathrm{~m}, 0 \mathrm{~m})$ in Cartesian coordinates. Two dipoles $\mathrm{P}_{1}$, $\mathrm{P}_{2}$ as receiving antennas, are placed at positions $(5 \mathrm{~m}, 0.4 \mathrm{~m})$ and $(6 \mathrm{~m},-0.2 \mathrm{~m})$, respectively. In order to build a multipath propagation environment, seventy-five perfect electrical conductivity (PEC) rods (0.1-m long and 0.04-m diameter) are set uniformly in the area $x \in[1,4]$ and $y \in[-0.4,0.4]$ between the radiation antenna and the receiving antennas. The schematic and CST implementation of the model are shown in Fig. 2 (a) and (b), respectively.

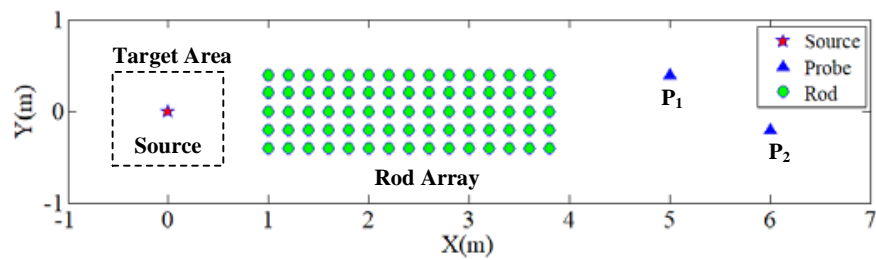

(a)

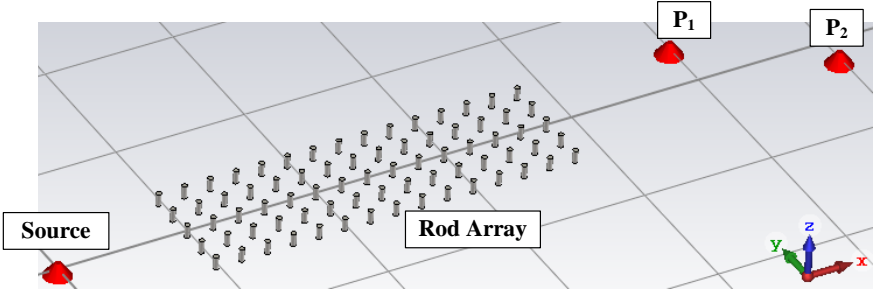

(b)

Fig. 2. The schematic (a) and CST model (b) of the considered configuration.

A modulated Gaussian pulse is considered as the excitation:

$$
s(t)=A_{0} \sin \left(2 \pi f_{0} t\right) e^{-4 \pi\left(\frac{t-\tau_{1}}{\tau_{2}}\right)^{2}}
$$

where $f_{0}$ is the center frequency, $\tau_{1}$ and $\tau_{2}$ are the parameters that regulate the bandwidth of the signal, and $A_{0}$ is the amplitude of the signal. It should be noted that the frequency range is set as $1 \mathrm{GHz}$ to $5 \mathrm{GHz}$. As shown in Fig. 3, the signals received by the receiving antennas are different from the point of view of their amplitude and waveform. 


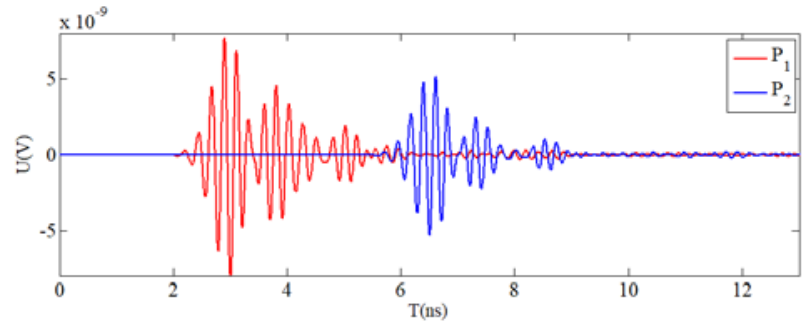

Fig. 3. Signals received by the receiving antennas. The positions of $P_{1}$ to $P_{2}$ are shown in Fig. 2.

The target area is chosen as $x \in[-0.5,0.5] y \in[-0.5,0.5]$ in the $\mathrm{z}=0$ plane, meshed into multiple points with a spacing of $0.1 \mathrm{~m}$, which are treated as the guessed source positions for the calculations. The $S_{12}$ parameters between the guessed source positions and the positions of receiving antennas, which are treated as transfer functions, are obtained based on the simulations. With the help of the Matrix Laboratory (MATLAB), we implemented the proposed method and evaluated the correlations at each point in the considered target area based on the steps mentioned before. The calculation frequency band was chosen to be the same as the frequency range of the excitation signal. Figs. 4(a) and 4(b) show the correlation coefficient distribution by using either the magnitude or the phase correlation criteria. The maximum coefficient identified by both criteria occurred at $(1 \mathrm{~m}, 0.3 \mathrm{~m})$, which corresponds to the position of the radiation source, represented by a black circle.

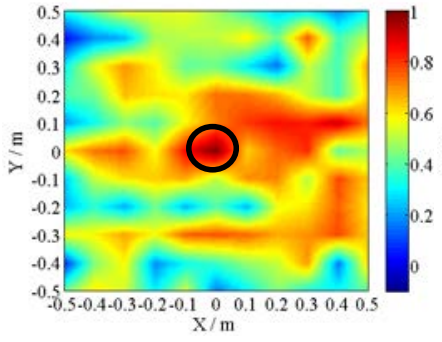

(a)

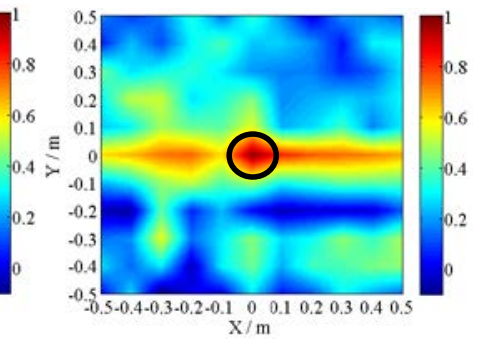

(b)
Fig. 4. The correlation coefficient distribution. (a) Magnitude Correlation Criterion; (b) Phase Correlation Criterion.

\section{Simplified Propagation Model in a Reflective ENVIRONMENT}

The simulations presented in the previous section show that the EMTR technique with the correlation criterion can be deployed to locate sources in a multipath environment using CST-MWS full-wave simulations for the backward procedure. However, the use of a full-wave simulation tool is non-ergodic and can be costly in terms of computer resources and simulation time. Therefore, a simplified propagation model based on the ray-tracing method is presented in this section to efficiently calculate transfer functions as a function of the reflection coefficient at the walls of a model of an indoor environment. In the first subsection (III.A), expressions for the electric field in the frequency domain as a function of the reflection coefficient are derived using the ray tracing method. In the second subsection (III.B), approximate time domain expressions for the reflection coefficient, based on expressions in the literature, are derived. These time domain expressions will be numerically transformed into the frequency domain when we apply the simplified propagation model derived in this section to implement the proposed correlation-based technique in Section IV and V.

\section{A. Multipath Propagation Modeling}

The analysis in Section II shows that obtaining the transfer functions between the observation point and the given guessed source positions is important in the proposed criterion. The transfer functions can be determined in advance, either through experiments or full-wave numerical simulations. However, the process can be rather time-consuming and non-ergodic. To cope with these issues, approximate numerical simulations can be used to compute the transfer functions. Although the calculation is an approximation to the realistic electromagnetic wave propagation, the numerical method is widely accepted and validated when its application conditions are met. For example, based on the geometrical optics theory, the ray tracing method is frequently used to predict the impulse response of the radio channel in indoor environments [35-36].

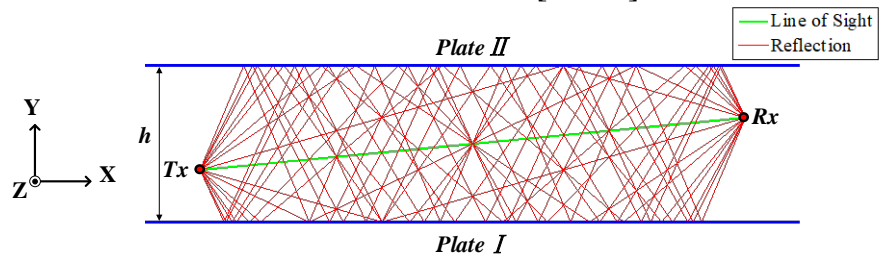

Fig. 5. Parallel plate waveguide. LOS wave and multiple reflections are modeled by using the ray tracing technique.

As a simplified example, the indoor environment can be treated as a parallel plate waveguide (PPW) with two plates around the source $T x$ and receiver $R x$ [24]. The example is considered to illustrate the approach. The plates are assumed to be made of the same material with a relative dielectric constant $\varepsilon_{r}$ and an electrical conductivity $\sigma$. As shown in Fig. 5, the received signal results from the multiple reflections (shown in red in Fig. 5) off the two plates, along with the LOS wave, shown in green [37-38]. Note that the environment is treated here as a two-dimensional (2D) model, but the approach could be readily extended to the three-dimensional space (3D) by using the Tent law [39]. The length of the plates along the $x$ and $z$ axes is assumed to be infinite and the plate separation along the $y$ axis is $l_{0}$. It is reasonable to assume specular reflection for all the multiple reflections in such a scenario, because it is reasonable to assume $l_{0}$ as being much larger than the wavelength of the signals [40]. Under that condition, the ray tracing approach, which is characterized by a good compromise between accuracy and ease of implementation, can be used to model the propagation process [41].

Based on the image theory, all the rays coming from $T x$, which would be received after being reflected by the surfaces, can be divided into the reflections from plate I and the reflections from plate II. Both cases follow common laws. Take the reflections coming from plate I as an example. As illustrated in Fig. 6, Rays 1 and 2, come, respectively, from images $T x^{1}$ and $T x^{2}$ as the first-order and second-order mirror source of 
$T x$, as viewed by the receiver $R x$.

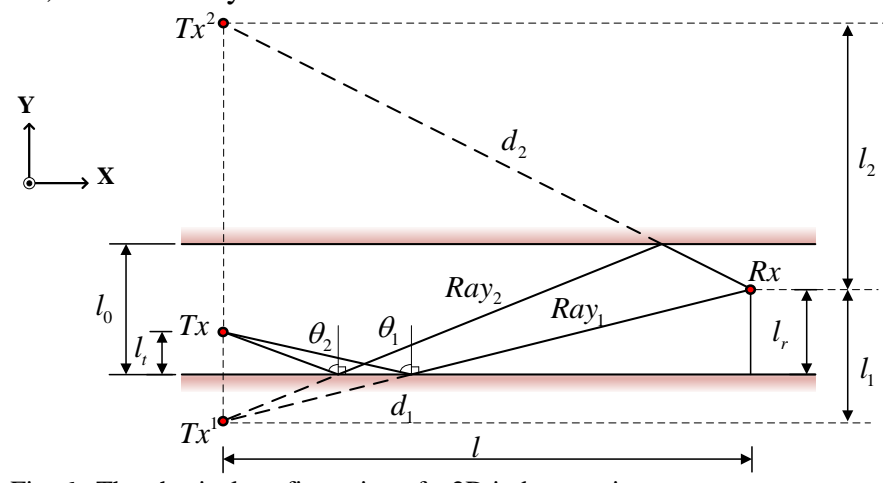

Fig. 6. The physical configuration of a 2D indoor environment.

The electric field arriving at the measuring point $E_{\text {total }}(f)$ is composed of LOS wave $E_{\text {Direct }}(f)$ and reflected wave (NLOS paths) $E_{\text {Reflected }}(f)$,

$$
E_{\text {total }}(f)=E_{\text {Direct }}(f)+E_{\text {Reflected }}(f)
$$

For the LOS path,

$$
E_{\text {Direct }}(f)=\frac{S(f)}{d} e^{-j k d}
$$

where $S(f)$ is the electric field from the source measured at the receiver, $d$ is the distance between the source and the receiver and $k$ is the wave number [32].

The overall NLOS wave can be expressed as

$$
E_{\text {Reflected }}(f)=\sum_{n=1}^{N}\left[\Gamma\left(\theta_{n}\right)\right]^{n} \frac{S(f)}{d_{n}} e^{-j k d_{n}}
$$

where $n$ is the order of the reflection, corresponding to the reflection coefficient $\Gamma\left(\theta_{n}\right)$ with the reflection angle $\theta_{n}$. As shown in Fig. 6, $\theta_{n}$ can be obtained by the triangle relationship

$$
\theta_{n}=\arcsin \left(\frac{l}{d_{n}}\right)
$$

where $l$ is the horizontal distance between the source and the receiver. Note that $d_{n}=\sqrt{l_{n}^{2}+l^{2}}$, where $l_{n}$ is the vertical distance between the $n$-order image $T x^{n}$ and the receiver. Thus, $l_{n}$ can be calculated by the following equation:

$$
l_{n}=l_{t}-(-1)^{n} l_{r}+n \cdot l_{0}-\left(1-(-1)^{n}\right) l_{0} / 2
$$

where $l_{t}$ and $l_{r}$ are the distances between $T x$ and plate I, and between $R x$ and plate I, respectively.

Therefore, the electric field at the receiver position can be expressed as:

$$
E_{\text {total }}(f)=E_{\text {Direct }}(f)+E_{\text {Reffected }}(f)=\sum_{n=0}^{N}\left[\Gamma\left(\theta_{n}\right)\right]^{n} \frac{S(f)}{d_{n}} e^{-j k d_{n}}
$$

From (19), $E(f)$ can be expressed as

$$
E_{\text {total }}(f)=S(f) \cdot\left\{\sum_{n=0}^{N} \frac{\cos k d_{n}}{d_{n}}\left[\Gamma\left(\theta_{n}\right)\right]^{n}-j \sum_{n=0}^{N} \frac{\sin k d_{n}}{d_{n}}\left[\Gamma\left(\theta_{n}\right)\right]^{n}\right\}
$$

Thus, the magnitude $\left|E_{\text {total }}(f)\right|$ and phase $\varphi_{\text {total }}(f)$ of the electric field can be expressed as follows:

$$
\begin{gathered}
\left|E_{\text {toual }}(f)\right|=S(f) \cdot \sqrt{\left\{\left[\sum_{n=0}^{N} \frac{\cos k d_{n}}{d_{n}}\left[\Gamma\left(\theta_{n}\right)\right]^{n}\right]^{2}+\left[\sum_{n=0}^{N} \frac{\sin k d_{n}}{d_{n}}\left[\Gamma\left(\theta_{n}\right)\right]^{n}\right]^{2}\right\}} \\
=S(f) \cdot \sqrt{\left[\sum_{n=0}^{N} \frac{\left[\Gamma\left(\theta_{n}\right)\right]^{2 n}}{d_{n}^{2}}+2 \cdot \sum_{\substack{n_{n}=0 \\
n_{2}=n_{n}}}^{N} \frac{\left[\Gamma\left(\theta_{n_{1}}\right)\right]^{n_{n}} \cdot\left[\Gamma\left(\theta_{n_{2}}\right)\right]_{2}^{n_{2}}}{d_{m_{1}} \cdot d_{n_{2}}} \cos \left(k d_{n_{1}}-k d_{n_{2}}\right)\right\}} \\
\varphi_{\text {total }}(f)=\arctan \frac{\sum_{n=0}^{N} \frac{\sin k d_{n}}{d_{n}}\left[\Gamma\left(\theta_{n}\right)\right]^{n}}{\sum_{n=0}^{N} \frac{\cos k d_{n}}{d_{n}}\left[\Gamma\left(\theta_{n}\right)\right]^{n}}
\end{gathered}
$$

As can be seen from (22) and (23), the magnitude and phase of the electric field are expressed in terms of $\Gamma\left(\theta_{n}\right)$. In the next subsection, based on a time domain expression available in the literature, an approximate time domain expression $h(t)$ for the reflection coefficient $\Gamma\left(\theta_{n}\right)$ will be derived. As explained earlier, the approximate time domain expression will be converted into the frequency domain when the correlation-based EMTR method is applied in Section IV.

\section{B. Approximate Expression for the Reflection Coefficient in} the Time Domain

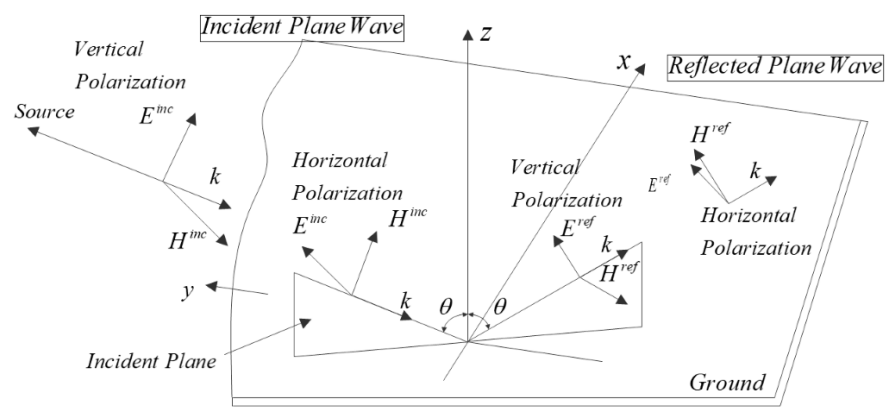

Fig. 7. Incident and reflected plane wave field in lossy half space.

As shown in Fig. 7, when either a vertically or a horizontally polarized incident plane wave is impinging on a finitely conducting half-space with a vertical angle of incidence $\theta$, the first-order signal reflected by the plane is usually composed of the undistorted and distorted terms [42]. The impulse response, which is the time domain counterpart of the reflection coefficient, is expressed as:

$$
h(t)= \pm\left(K \delta(t)+\frac{4 \kappa}{1-\kappa^{2}} e^{-a t} \Psi\right)
$$

where $K$ is the proportion coefficient shown as $K=\frac{1-\kappa}{1+\kappa}$ with $\kappa=\frac{\sqrt{\varepsilon_{r}-\sin ^{2} \theta}}{\varepsilon_{r} \cos \theta}$ for vertical polarization and $\kappa=\frac{\cos \theta}{\sqrt{\varepsilon_{r}-\sin ^{2} \theta}}$ for horizontal polarization, and the \pm sign also depends on the polarization. Here, $\varepsilon_{r}$ and $\sigma$ represent the relative permittivity and the electrical conductivity of the reflection surface, respectively, $c$ is the speed of light, and 
$a=\frac{60 \pi \sigma c}{\varepsilon_{r}}$. In addition, $\Psi$ represents the Bessel Function.

The total E-field can be composed of horizontally and vertically polarized components. In the present model, the horizontal components for both line-of-sight and reflected EM waves are directed along the z-axis. However, the vertical E-fields are decided by the reflection order due to the incident angle. Thus, it is meaningful to take only the horizontal polarization incidence into consideration.

In order to apply the expression above to practical problems, it is desirable to use an approximate expression for the Bessel function. Based on [43], the Bessel functions can be expressed as $\Psi=\sum_{k=0}^{\infty} \Phi_{k}$, where

$$
\begin{aligned}
\Phi_{k}= & \frac{1}{2 K(k !)}\left(\frac{x}{K^{2}}\right)^{k-1}(-1)^{k} \\
& \times\left\{(x+k)\left[e^{-x}-\sum_{m=0}^{k} \frac{(-1)^{m} x^{m}}{m !}\right]+\frac{(-1)^{k} x^{k+1}}{k !}\right\}
\end{aligned}
$$

where $x=\frac{K a t}{2}$.

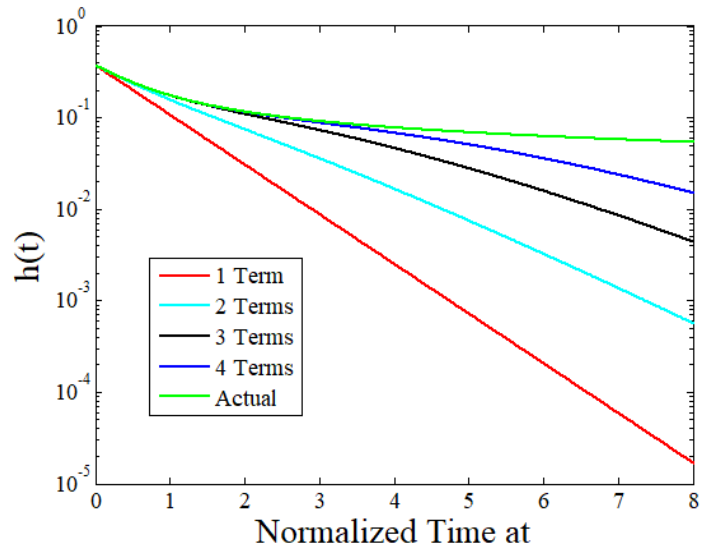

Fig. 8. Total and partial sums for the reflected field impulse response for $\theta=45^{\circ}, \varepsilon_{\mathrm{r}}=5$, and $\sigma=0.1$ with horizontal polarization.

The relative permittivity and the conductivity of the plate are assumed to be 5 and $0.1 \mathrm{~S} / \mathrm{m}$, respectively, which are representative of common interfaces (ground, wall and ceiling) found in buildings or urban environments. Let us consider an angle $\theta=45^{\circ}$ as an example. The first-order results calculated by the actual and approximate functions are shown in Fig. 8. The curves labeled as " 1 term" , "2 Terms", "3 Terms" and "4 Terms" result from approximating $\Psi$ by $\Phi_{0}, \Phi_{0}+\Phi_{1}$, $\Phi_{0}+\Phi_{1}+\Phi_{2}$ and $\Phi_{0}+\Phi_{1}+\Phi_{2}+\Phi_{3}$. When at $\leq 2$, retaining the first three terms $\Phi_{0}+\Phi_{1}+\Phi_{2}$ is sufficient to approximate $h(t)$ for the early-time response. For the considered permittivity and electrical conductivity, this corresponds to $t \leq 1.768 \mathrm{~ns}$. Thus, the expression for the impulse response can be expressed as follows:

$$
h(t) \approx-\left[K \delta(t)+\frac{4 \kappa}{1-\kappa^{2}} e^{-a t} \cdot\left(\Phi_{0}+\Phi_{1}+\Phi_{2}\right)\right]
$$

In order to meet the condition that the pulse duration time $t \leq 1.768 \mathrm{~ns}$, the central frequency and the frequency range are set to $2.7 \mathrm{GHz}$ and $0.3 \mathrm{GHz}-5 \mathrm{GHz}$, respectively. The resulting pulse duration is $0.8 \mathrm{~ns}$. The polarized direction is chosen to be along the z-axis. Fig. 9(a) shows the waveforms of the incident and the reflected signal from the plates, including the undistorted term and the distorted term as expressed in (24). In order to show the distorted term clearly, an expanded view of the distorted term is shown in Fig. 9(b).

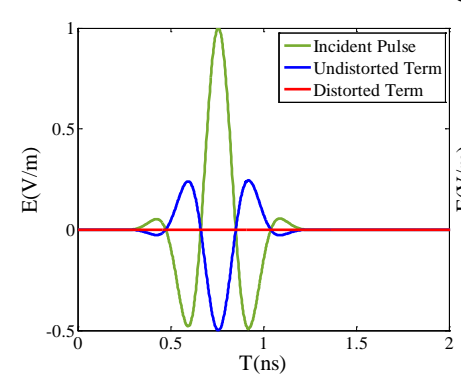

(a)

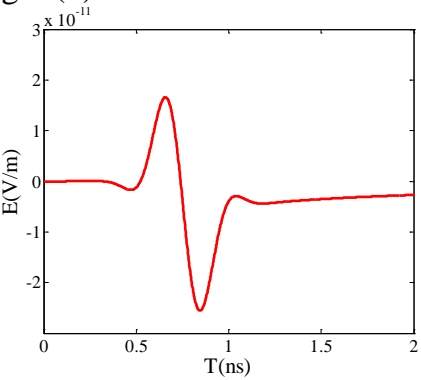

(b)
Fig. 9. (a) The pulse signal and its reflection by a half- space. The reflected signal is the sum of the distorted and undistorted terms defined in Equation (21). (b) Expanded view of the distorted term.

The numerical results of the reflected signal are consistent with the results presented in [43]. Combined with Equation (26), it can be found that the distorted term is much smaller than the undistorted term for any angle of incidence. Therefore, in the case mentioned above, the distorted term can be neglected in the calculation of the first-order reflection, and the impulse response can be expressed as:

$$
h(t)=-K \delta(t)=-\frac{1-\kappa}{1+\kappa} \delta(t)=-\frac{1-\frac{\cos \theta}{\sqrt{\varepsilon_{r}-\sin ^{2} \theta}}}{1+\frac{\cos \theta}{\sqrt{\varepsilon_{r}-\sin ^{2} \theta}}} \delta(t)
$$

The approximated impulse response will be transformed to the frequency domain and applied to simulations in the next section in order to calculate the overall electric field.

Based on the derivation presented in this section, we will demonstrate in Section IV an application of the proposed source localization method to a case study. We then analyze the performance of the correlation criterion by building the simplified model in simulation. Section $\mathrm{V}$ assesses the performance of the proposed method in practical applications by using a full-wave numerical simulation in the forward procedure and the ray tracing method in the backward phase.

\section{Performance Assessment of The Correlation CRITERIA TO LOCATE TRANSIENT SOURCES}

In this section, numerical simulations are carried out to investigate the relationship between the spatial resolution of the proposed algorithm based on either the magnitude or the phase correlation criterion and the effect of influencing factors including the central frequency and the bandwidth of the excitation source, the noise level, the baseline length, and the number of observation points. 


\section{A. General Description}

As in Section III, we will consider a 2D model, shown in Fig. 10 with two plates located at $y=0$ and $y=0.8 \mathrm{~m}$, respectively, in Cartesian coordinates. The permittivity and conductivity of the plates are respectively 5 and $0.1 \mathrm{~S} / \mathrm{m}$. The source is set at (1 $\mathrm{m}, 0.3 \mathrm{~m}$ ). The polarization of the E-field in the far field region is perpendicular to the $x-y$ plane. Four E-field probes $P_{1}$ to $P_{4}$, placed at $(7 \mathrm{~m}, 0.6 \mathrm{~m}),(8 \mathrm{~m}, 0.5 \mathrm{~m}),(9 \mathrm{~m}, 0.4 \mathrm{~m})$ and $(10 \mathrm{~m}, 0.3$ $\mathrm{m})$, respectively, form a time reversal mirror (TRM) array. In order to improve the calculation efficiency, the target area is chosen as a rectangle with $x \in[-1,3]$ and $y \in[0.1,0.7]$ in the $z$ $=0$ plane. Both the forward and backward procedures are numerically calculated in the MATLAB environment.

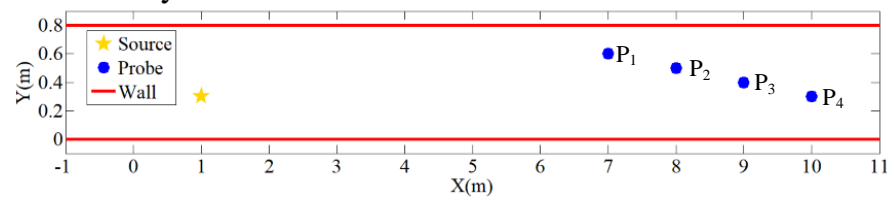

Fig. 10. Top view of propagation model.

The waveform of the excitation is chosen to be a modulated Gaussian pulse in the form of (14). In the first example, $f_{0}=2.7 \mathrm{GHz}$, and the $-20 \mathrm{~dB}$ frequency range is set as 0.3 to $5 \mathrm{GHz}$ by adjusting $\tau_{1}$ and $\tau_{2}$. The noise is assumed to be zero. Even though there are an infinite number of paths from the source to each probe, as opposed to the case of a cavity with highly reflective walls [24], only a few of the paths have meaningful contributions because the energy of the signal decreases very rapidly due to the energy loss caused by reflection and free space propagation [40]. Based on the considered electrical parameters for the two plates, the maximum number of reflection paths is limited to 10 .

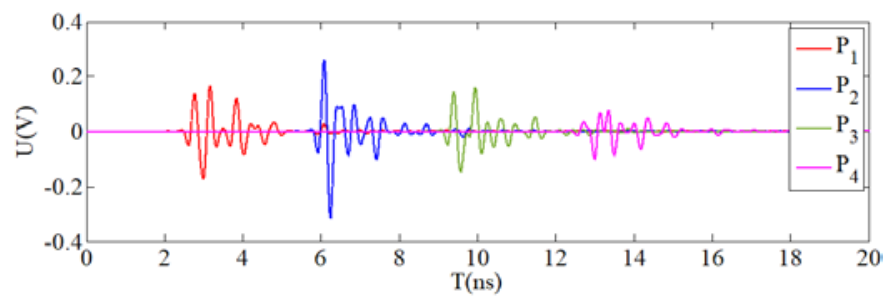

Fig. 11. Signals received by the probes. The positions of $\mathrm{P}_{1}$ to $\mathrm{P}_{4}$ are shown in Fig. 10.

The forward procedure is carried out using the propagation model derived in Section III in which the time domain reflection coefficient given by Equation (27) has been converted into the frequency domain using the FFT. As shown in Fig. 11, the signals received by all the probes are different from the point of view of their amplitude and waveform.

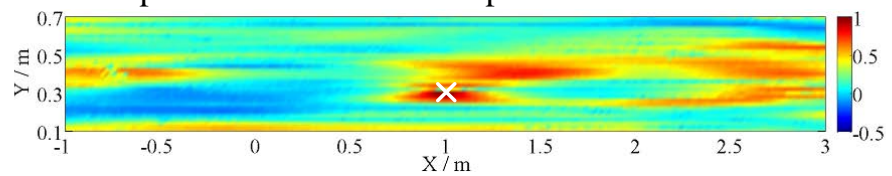

(a)

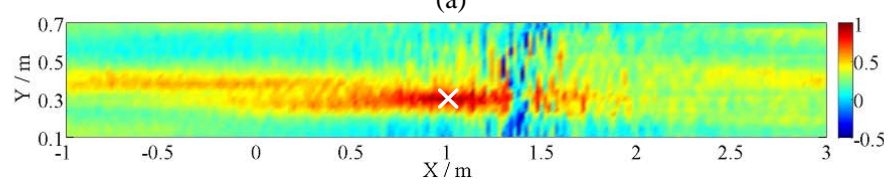

(b)

Fig. 12. The correlation coefficient distribution. (a) Magnitude Correlation Criterion; (b) Phase Correlation Criterion.

Following the steps described in Section II, we applied the proposed method in the backward procedure and evaluated the correlations at each point in the considered target area. The signals measured at $\mathrm{P}_{1}$ and $\mathrm{P}_{4}$ (see Fig. 10) were selected to calculate the correlation distribution. The calculation frequency band was chosen to be the same as the $-20 \mathrm{~dB}$ frequency band of the excitation signal. Figs. 12(a) and 12(b) show the correlation coefficient distribution by using, respectively, either the magnitude or the phase correlation criterion. Represented by a white cross, the maximum coefficient identified by both criteria occurred at $(1 \mathrm{~m}, 0.3 \mathrm{~m})$, which corresponds to the position of the radiation source. It is clear from the simulations that the resolution of the magnitude correlation criterion is better than that of the phase criterion. Furthermore, it can be found from Equation (22) that the magnitude correlation criterion will not be affected by the time synchronization errors between the observation points once the time length of the recording is sufficient, since the error would be cancelled by the term $\cos \left(k r_{n_{1}}-k r_{n_{2}}\right)$.

\section{B. Influence of the Bandwidth}

The effect of the excitation bandwidth on the spatial resolution is investigated by varying $\tau_{1}, \tau_{2}$ and $f_{0}$. The results in Fig. 13 and Fig. 14 show that the spatial resolution increases as the bandwidth of the excitation increases, which means that excitations with a wider bandwidth would result in a better spatial resolution.

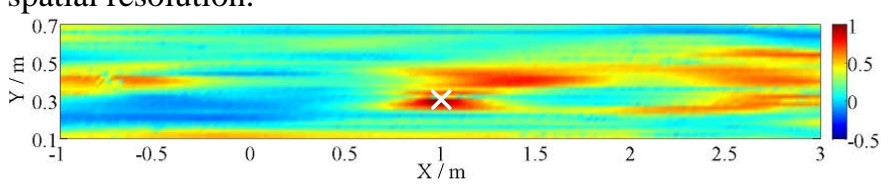

(a)

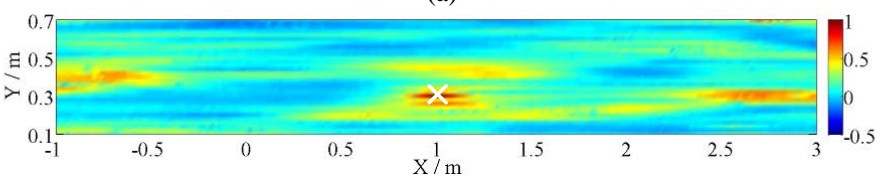

(b)

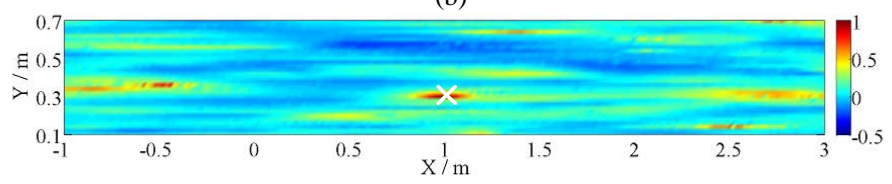

(c)

Fig. 13. Spatial resolution obtained using the magnitude correlation criterion for three different bandwidths with lower cutoff frequency $0.3 \mathrm{GHz}$ : (a) 4.7 GHz; (b) $6.7 \mathrm{GHz}$; (c) $8.7 \mathrm{GHz}$.

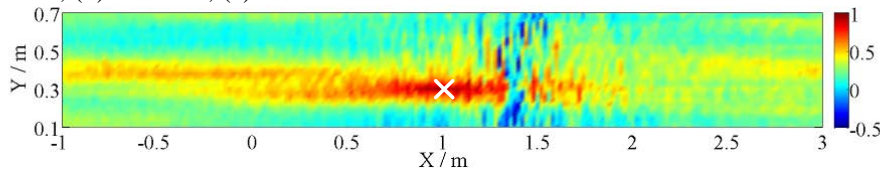

(a)

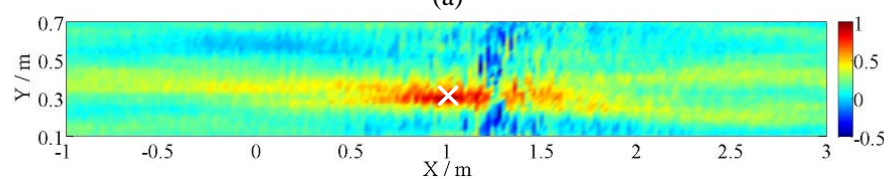

(b) 


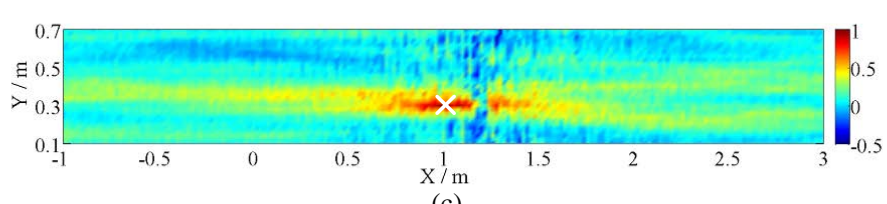

(c)

Fig. 14. Spatial resolution obtained using the phase correlation criterion for three different bandwidths with lower cutoff frequency $0.3 \mathrm{GHz}$ : (a) $4.7 \mathrm{GHz}$; (b) $6.7 \mathrm{GHz}$; (c) $8.7 \mathrm{GHz}$.

\section{Influence of the Central Frequency}

The results in Fig. 15 and Fig. 16 show the spatial resolution as a function of the central frequency of the excitation. It is clear that, as the central frequency of the excitation increases, the spatial resolution increases, meaning excitations with a higher central frequency would result in a better spatial resolution. However, for the magnitude correlation criterion, the side lobes would also increase as the central frequency increases, which may degrade the robustness of the method against noise.

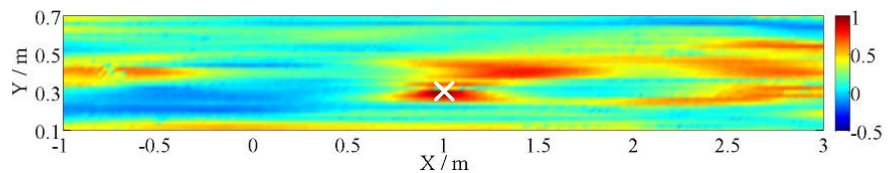

(a)

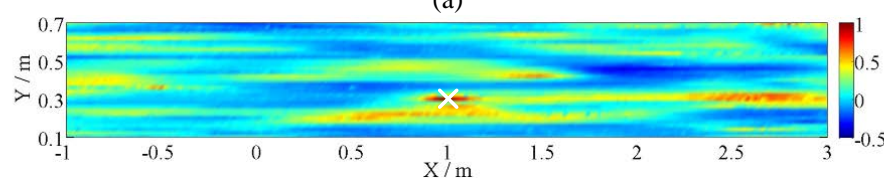

(b)

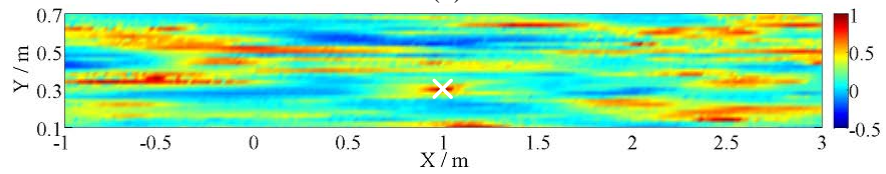

(c)

Fig. 15. Spatial resolution obtained using the magnitude correlation criterion for three different central frequencies with bandwidth $4.7 \mathrm{GHz}$ : (a) $2.7 \mathrm{GHz}$; (b) 4.7 GHz; (c) $6.7 \mathrm{GHz}$.

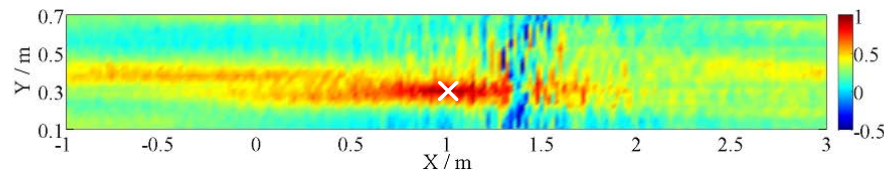

(a)

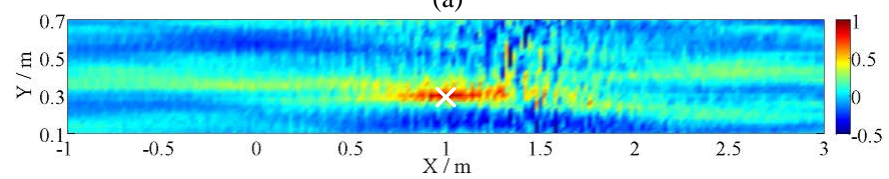

(b)

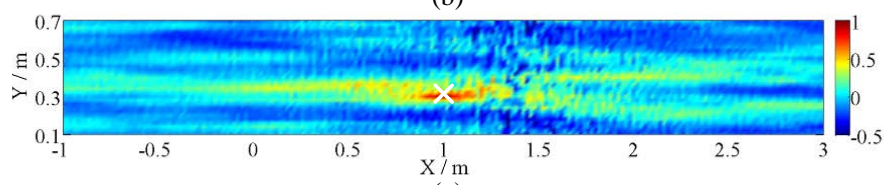

(c)

Fig. 16. Spatial resolution obtained using the phase correlation criterion for three different central frequencies with bandwidth $4.7 \mathrm{GHz}$ : (a) $2.7 \mathrm{GHz}$; (b) 4.7 GHz; (c) $6.7 \mathrm{GHz}$.

\section{Influence of the Noise Level}

In this section, we assess the robustness of the method with respect to noise. Since the noise caused by the measurement system is of primary concern, a white Gaussian noise of equivalent intensity is added to the signals measured by each probe in sections IV (B) and (C). The probability density of the noise is expressed as

$$
P(x)=\frac{1}{\sqrt{2 \pi \cdot \operatorname{Var}}} \exp \left(-\frac{(x-\mu)^{2}}{2 \cdot \operatorname{Var}}\right)
$$

where $\mu$ is the mean value of the noise and Var is the variance which represents the noise power density.

In the simulation, $\mu$ was set to zero and $\sigma^{2}$ in logarithmic form was varied from -6 to -1.5 . The results were derived from 100 independent trials, expressed by the Root Mean Square Error (RMSE) computed using

$$
R M S E=\sqrt{\frac{1}{100} \sum_{m=1}^{100}\left\|r_{m}-r_{s}\right\|^{2}}
$$

where $r_{m}$ is the $m$-th estimation result and $r_{s}$ is the position of the source.

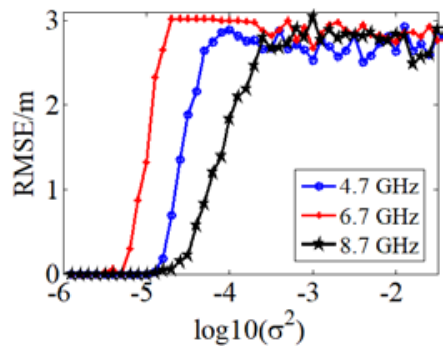

(a)

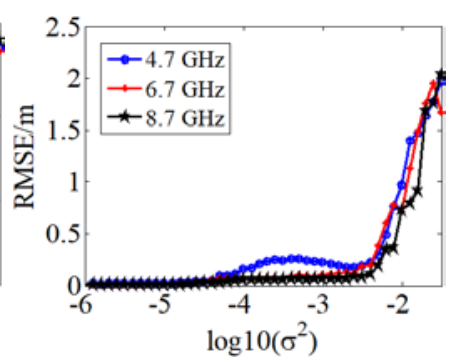

(b)
Fig. 17. Simulation results of RMSE versus $\sigma^{2}$ for different bandwidths: (a) magnitude correlation criterion and (b) phase correlation criterion.

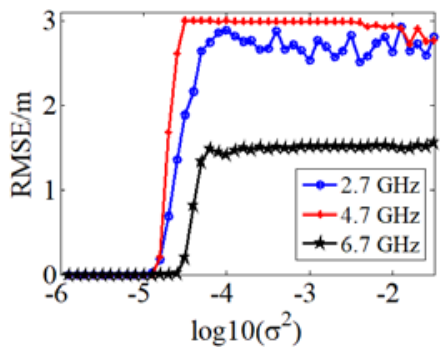

(a)

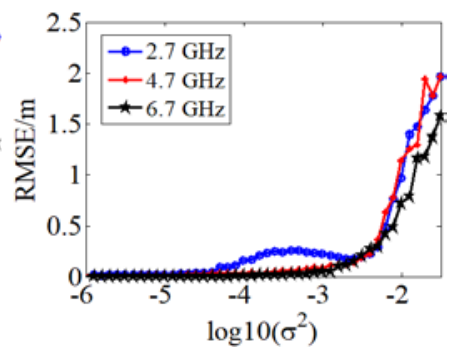

(b)
Fig. 18. Simulation results of RMSE versus $\sigma^{2}$ for different center frequencies: (a) magnitude correlation criterion and (b) phase correlation criterion.

As shown in Fig. 17 and Fig. 18, the method based on the phase correlation criterion behaves more robustly against noise than that based on the magnitude correlation. Furthermore, the anti-noise performance seems to be similar for signals with different central frequencies or bandwidths for the phase correlation criterion.

\section{E. Influence of the Baseline Length and the Number of Observation Points}

In order to study the influence of the number of observation points, we selected three kinds of combinations from the array in Fig. 10: (1) two elements, $\mathrm{P}_{1}, \mathrm{P}_{4}$, represented as L1; (2) two elements, $\mathrm{P}_{2}, \mathrm{P}_{3}$, represented as L2; (3) four elements, $\mathrm{P}_{1}, \mathrm{P}_{2}, \mathrm{P}_{3}$, $\mathrm{P}_{4}$, represented as L3. Since the Pearson correlation coefficient is only applicable to two variables, the result for L3 was 
obtained by multiplying together the three correlation coefficients acquired after applying the Pearson correlation formula to each pair of signals.

The results presented in Fig. 19 and Fig. 20 show that increasing the number of probes and the baseline length of the array would improve the spatial resolution of the position, which could help to detect and locate multiple sources with small spacings between them.

In addition, the cross-range resolution is better than the down-range one for both, the magnitude and the phase correlation distributions. Indeed, the effective aperture of the array in the down-range is smaller than that in the cross-range, where multiple mirror sources are taken into consideration due to reflections, which increases the effective aperture in the cross-range.

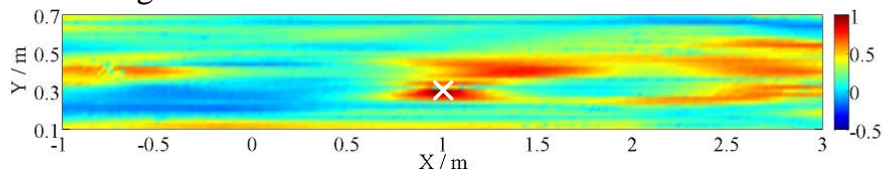

(a)

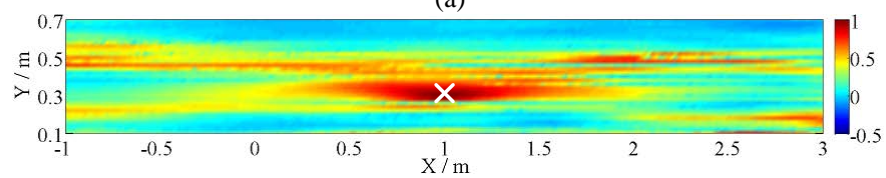

(b)

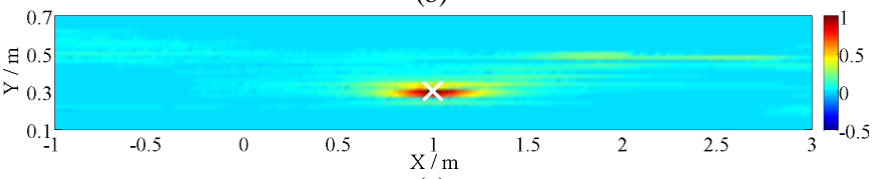

(c)

Fig. 19. Spatial resolution obtained using the magnitude correlation criterion for: (a) L1, (b) L2, (c) L3.

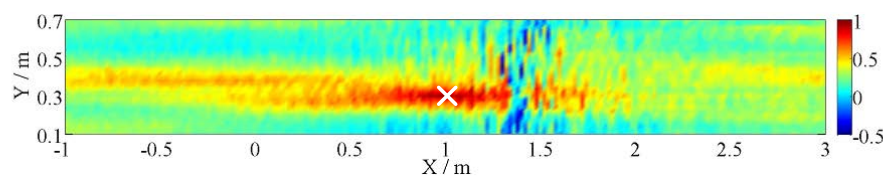

(a)

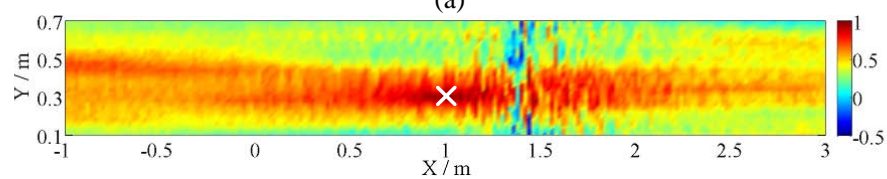

(b)

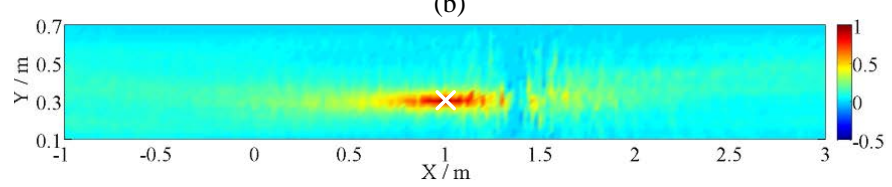

(c)

Fig. 20. Spatial resolution obtained using phase correlation criterion for: (a) L1, (b) L2, (c) L3.

\section{Validation of The Proposed Correlation Method to LOCATE SOURCES}

In order to test the performance of the proposed method in practical applications, the location of the source is estimated in this section using full-wave numerical simulations for the direct time within CST-MWS and using the proposed ray tracing method for the backward procedure within MATLAB. It is worth noting that the back-propagation step of the proposed method could not be simulated in CST-MWS or in other commercial EM simulation software because they do not allow the implementation of inverted-loss model parameters.

As shown in Fig. 21(a), a propagation model was established according to the considered configuration in Sections III and IV. A dipole, located at position $(1.0 \mathrm{~m}, 0.3 \mathrm{~m})$ in Cartesian coordinates, was used as the emitter, the direction of which was chosen to be along the z-axis. In order to simulate the plates, two cuboids, made of a material with a relative permittivity $\varepsilon=5$ and a conductivity $\sigma=0.1 \mathrm{~S} / \mathrm{m}$, were set at $y=0$ and $y=0.8$, parallel to the $x$-z plane. The length and width of the cuboids were set to be significantly larger than the antenna. The excitation signal was chosen to be a Gaussian pulse signal with $-20 \mathrm{~dB}$ bandwidth of $1 \mathrm{GHz}-5 \mathrm{GHz}$, in order to have, as much as possible, the plates in the far field region of the radiation antenna. An array consisting of two probes referred to as $\mathrm{P}_{1}$ and $\mathrm{P}_{2}$, located at $(8.0 \mathrm{~m}, 0.5 \mathrm{~m})$ and $(9.0 \mathrm{~m}, 0.3 \mathrm{~m})$, respectively, was used to measure the z-axis polarized E-field. As a result of the considered polarization, the derived approximation for the reflection function can be used in the localization. In order to improve the computation efficiency, the dipole and probes were assumed to be at the same coordinate in the z-axis, so that the model could be treated as 2D. A screenshot of the configuration within CST-MWS is shown in Fig. 21(b).

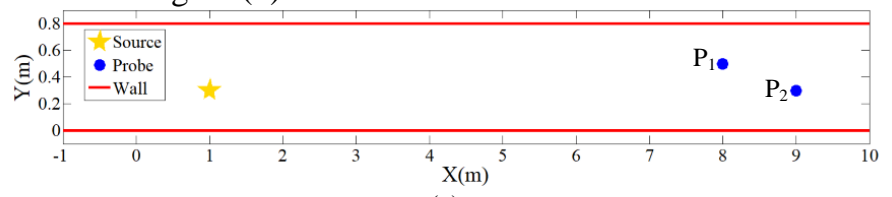

(a)

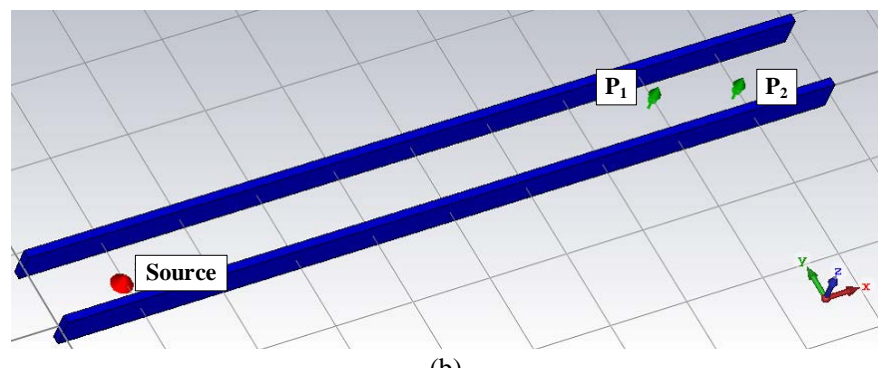

(b)

Fig. 21. Schematic (a) and CST-MWS implementation (b) of the numerical model.

Fig. 22(a) shows the resulting electric field waveforms at $\mathrm{P}_{1}$ and $\mathrm{P}_{2}$ within a time window of 14 ns. Due to multiple reflections from the two plates, the signal widths were increased in the time domain, which is the main reason for localization errors in the common approaches using the time difference of arrival method, since it is difficult to distinguish the LOS wave. 


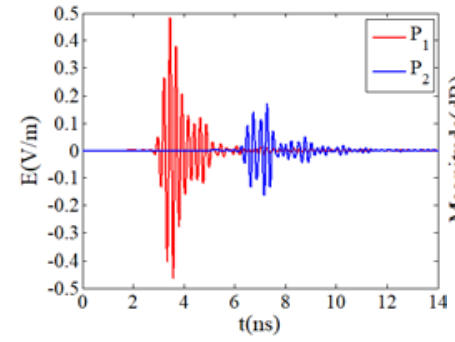

(a)

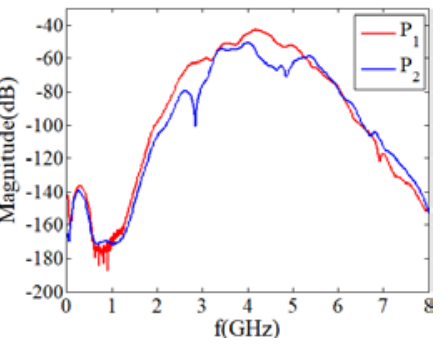

(b)
Fig. 22. Electric field waveforms measured by the two probes in the time domain (a) and their magnitude Fourier spectrum obtained using FFT (b).

Based on the measured signals and the positions of the measurement points, the proposed algorithm was employed to locate the source. The $-20 \mathrm{~dB}$ bandwidth of the received signals, namely [2.5 GHz, $5.5 \mathrm{GHz}$ ] was chosen to be calculation frequency band, as Fig. 22 (b) shows, since the components within this frequency band are dominant in those signals. Furthermore, the target area was chosen as a rectangular area with $x \in[-1,5]$ and $y \in[0.1,0.7]$ in the $z=0$ plane, meshed into multiple points with a spacing of $0.02 \mathrm{~m}$ which were treated as the guessed source positions for the calculations.

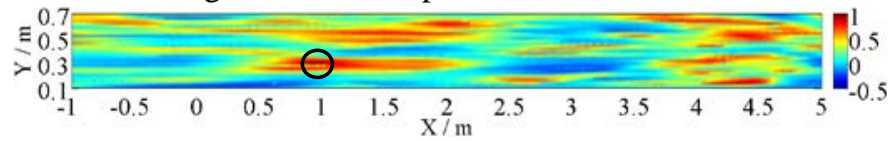

(a)

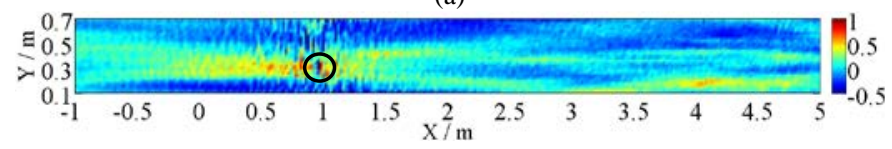

(b)

Fig. 23. Correlation distribution for the magnitude (a), and phase (b) in the target area.

As shown in Fig. 23, the correlation distributions were obtained and the maximum correlation position is represented by a black circle. The correlation coefficient for the magnitude reached its maximum at $(0.96 \mathrm{~m}, 0.30 \mathrm{~m})$, which corresponds to a position error of $0.04 \mathrm{~m}$. A tightly focused image was obtained with a maximum amplitude 0.9555 while the coefficients associated with most of the other points in the target area were generally lower than 0.9 . On the other hand, the maximum of the phase correlation coefficient occurred at ( $0.98 \mathrm{~m}, 0.32 \mathrm{~m})$, which corresponds to a position error of one mesh-cell $(0.02 \mathrm{~m})$ both in the cross range and in the down range. Specifically, the maximum phase correlation coefficient was 0.9595 , while the coefficients associated with most of the other points in the target area were lower than 0.5 and even negative.

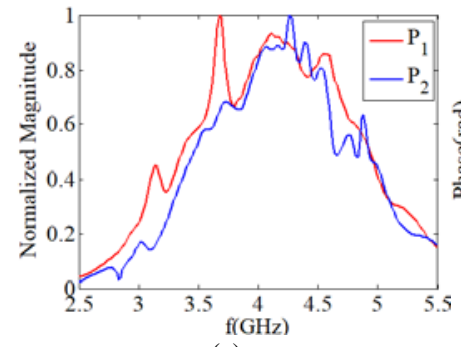

(a)

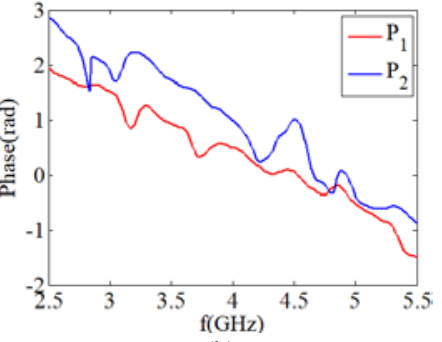

(b)
Fig. 24. The normalized magnitude (a) and phase (b) of the back-propagated z-axis polarized E-field signals at the maximum correlation coefficient position from measurement points $\mathrm{P}_{1}$ and $\mathrm{P}_{2}$.

By using the ray tracing technique, the normalized magnitude and phase of the back-propagated z-axis polarized E-field signals corresponding to the maximum correlation coefficient position are shown in Fig. 24. It can be clearly seen that the two signals are not totally the same, since the ray tracing technique is just an approximation to the EM propagation in a real environment. The intensity of the time reversed signal at the location of the source decreases as the Green's functions in the forward and backward steps become less correlated [44]. In our study, the ray tracing technique used in the backward step was close enough to the full-wave simulation used in the forward step, so that a sufficiently high correlation coefficient was obtained at the approximate source position. This is due to the fact that the application conditions of the ray tracing approximation are satisfied in the considered model.

Fig. 25 shows the EMTR location results using the maximum amplitude criterion and the maximum energy criterion, which correspond to the absolute electric field and the space energy peak, respectively [45]. The position predicted by the maximum amplitude criterion and the maximum energy criterion is $(4.6 \mathrm{~m}, 0.16 \mathrm{~m})$ and $(5 \mathrm{~m}, 0.3 \mathrm{~m})$, corresponding to location errors as large as $3.6 \mathrm{~m}$ and $4 \mathrm{~m}$, respectively. Actually, the normalized distribution of the maximum amplitude criterion and the maximum energy criterion have local maximum values (represented by the red circle in Fig. 25) at positions $(1.04 \mathrm{~m}, 0.3 \mathrm{~m})$ and $(1.1 \mathrm{~m}, 0.3 \mathrm{~m})$, which are close to the source position. However, without a priori knowledge of source, the search needs to be performed in the whole area and the absolute maxima will be erroneously identified as the source locations. In this case, the EMTR method with the proposed criterion using the correlation of the back-propagated signals is more accurate than that with the maximum amplitude criterion or the maximum energy criterion.

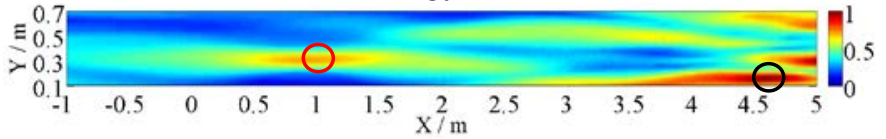

(a)

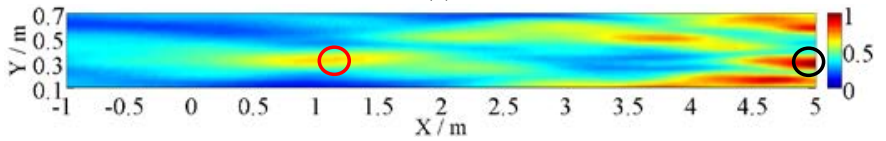

(b)

Fig. 25. The normalized distribution of the amplitude criterion (a) and the energy criterion (b) in the target area.

Although the proposed magnitude and phase correlation criteria were able to locate the source accurately in the considered cases, further studies, such as a frequency band optimization and an experimental verification for different scenarios, are needed before any definitive conclusions can be drawn concerning the location accuracy of the proposed method. 


\section{CONCLUSION}

In this paper, magnitude and phase correlation criteria were proposed to locate a transient EM radiation source in indoor reflective environments using the electromagnetic time reversal technique. Based on the results of numerical simulations, the following conclusions can be drawn.

1) The EMTR technique with the proposed criteria is able to accurately locate radiation sources in indoor environments by using only two observation points when the application conditions of the ray tracing method are met.

2) The anti-interference capability of the phase correlation criterion is better than that of the magnitude correlation criterion, while the magnitude correlation criterion is more suitable for scenarios where the two observation points are affected by time-alignment errors.

3) The spatial resolution of the localization could be slightly improved by using additional probes or by increasing the baseline length of the probes.

Work is in progress to investigate an optimal combination of the two criteria and the application of them to 3D structures.

\section{ACKNOWLEDGMENT}

The authors would like to give thanks to the High-Performance Computation Platform of Xi'an Jiaotong University for the support in the numerical simulation.

\section{REFERENCES}

[1] K. W. K. Lui and H. C. So, "Range-based source localisation with pure reflector in presence of multipath propagation," Electronics Letters, vol. 46, no. 13, pp. 957 -958, Jun. 2010.

[2] Joon-Yong Lee and Sungyul Yoo, "Large error performance of UWB ranging in multipath and multiuser environments," IEEE Transactions on Microwave Theory and Techniques, vol. 54, no. 4, pp. 1887-1895, Jun. 2006.

[3] G. V. Zaruba, M. Huber, F. A. Kamangar, and I. Chlamtac, "Indoor location tracking using RSSI readings from a single Wi-Fi access point," Wireless Network, vol. 13, no. 2, pp. 221-235, Apr. 2007.

[4] M. Scherhäufl, M. Pichler, E. Schimbäck, D. J. Müller, A. Ziroff and A. Stelzer, "Indoor Localization of Passive UHF RFID Tags Based on Phase-of-Arrival Evaluation,” IEEE Transactions on Microwave Theory and Techniques, vol. 61, no. 12, pp. 4724-4729, Dec. 2013.

[5] L. Huan and R. Bo, "Wireless location for indoor based on UWB," in 34th Chinese Control Conference (CCC), Jul. 2015, pp. 6430-6433.

[6] P. Setlur, G. E. Smith, F. Ahmad and M. G. Amin, "Target Localization with a Single Sensor via Multipath Exploitation,” IEEE Transactions on Aerospace and Electronic Systems, vol. 48, no. 3, pp. 1996-2014, Jul. 2012.

[7] D. Dardari, P. Closas and P. M. Djuric, "Indoor Tracking: Theory, Methods, and Technologies," IEEE Transactions on Aerospace and Electronic Systems, vol. 64, pp. 1263-1278, 2015.

[8] H. Miao, K. Yu and M. J. Juntti, "Positioning for NLOS Propagation: Algorithm Derivations and Cramer-Rao Bounds," IEEE Transactions on Vehicular Technology, vol. 56, no. 5, pp. 2568-2580, Sept. 2007.

[9] M. Bolic, M. Rostamian and P. M. Djuric, "Proximity Detection with RFID: A Step Toward the Internet of Things," IEEE Pervasive Computing, vol. 14, no. 2, pp. 70-76, Apr.-June 2015.

[10] Y. Wang, Y. Wu and Y. Shen, "Joint Spatiotemporal Multipath Mitigation in Large-Scale Array Localization," IEEE Transactions on Signal Processing, vol. 67, no. 3, pp. 783-797, 1 Feb.1, 2019.

[11] J. Wisanmongkol, L. Klinkusoom, T. Sanpechuda, L. Kovavisaruch and K. Kaemarungsi, "Multipath Mitigation for RSSI-Based Bluetooth Low Energy Localization," in 19th International Symposium on
Communications and Information Technologies (ISCIT), Sept. 2019, pp. 47-51.

[12] S. Al-Jazzar and J. Caffery, "ML and Bayesian TOA location estimators for NLOS environments," in Proceedings IEEE 56th Vehicular Technology Conference, Sept. 2002, pp. 1178-1181.

[13] S. Venkatraman and J. Caffery, "Hybrid TOA/AOA techniques for mobile location in non-line-of-sight environments," in IEEE Wireless Communications and Networking Conference (IEEE Cat. No.04TH8733), Mar. 2004, pp. 274-278.

[14] P. Setlur, M. Amin and F. Ahmad, "Multipath Model and Exploitation in Through-the-Wall and Urban Radar Sensing," IEEE Transactions on Geoscience and Remote Sensing, vol. 49, no. 10, pp. 4021-4034, Oct. 2011.

[15] P. Meissner and K. Witrisal, "Multipath-assisted single-anchor indoor localization in an office environment," in International Conference on Systems, Signals and Image Processing (IWSSIP), Apr. 2012, pp. 22-25.

[16] P. Meissner, D. Arnitz, T. Gigl and K. Witrisal, "Analysis of an indoor UWB channel for multipath-aided localization," in 2011 IEEE International Conference on Ultra-Wideband (ICUWB), Sept. 2011, pp. 565-569.

[17] M. Porretta et al., "A novel single base station location technique for microcellular wireless networks: description and validation by a deterministic propagation model,” IEEE Transactions on Vehicular Technology, vol. 53, no. 5, pp. 1502-1514, Sept. 2004.

[18] M. Porretta et al., "A novel single base station mobile location algorithm: description and validation using a deterministic propagation model," in 4th International Conference on 3G Mobile Communication Technologies, Jun. 2003, pp. 277-281.

[19] A. Abbasi and M. H. Kahaei, "Improving source localization in LOS and NLOS multipath environments for UWB signals," in 14th International CSI Computer Conference, Oct. 2009, pp. 310-316.

[20] J. L. Krolik, J. Farrell and A. Steinhardt, "Exploiting multipath propagation for GMTI in urban environments," in IEEE Conference on Radar, Apr. 2006, pp. 1-4.

[21] P. Kosmas and C. M. Rappaport, "Time reversal with the FDTD method for microwave breast cancer detection," IEEE Transactions on Microwave Theory and Techniques, vol. 53, no. 7, pp. 2317-2323, Jul. 2005.

[22] W. J. R. Hoefer, "Computational Time Reversal—A Frontier in Electromagnetic Structure Synthesis and Design," IEEE Transactions on Microwave Theory and Techniques, vol. 63, no. 1, pp. 3-10, Jan. 2015.

[23] S. Mukherjee, L. Udpa, S. Udpa, E. J. Rothwell and Y. Deng, "A Time Reversal-Based Microwave Imaging System for Detection of Breast Tumors," IEEE Transactions on Microwave Theory and Techniques, vol. 67, no. 5, pp. 2062-2075, May. 2019.

[24] H. Karami, M. Azadifar, A. Mostajabi, P. Favrat, M. Rubinstein and F. Rachidi, "Localization of Electromagnetic Interference Sources Using a Time Reversal Cavity,” IEEE Transactions on Industrial Electronics, early access, Jan. 2020, doi: 10.1109/TIE.2019.2962460.

[25] M. Davy, J. de Rosny and M. Fink, "Focusing and amplification of electromagnetic waves by time-reversal in an leaky reverberation chamber," in 2009 IEEE Antennas and Propagation Society International Symposium, Jul. 2009, pp. 1-4.

[26] L. Li and J. L. Krolik, "Simultaneous Target and Multipath Positioning," IEEE Journal of Selected Topics in Signal Processing, vol. 8, no. 1, pp. 153-165, Feb. 2014.

[27] H. Ding, W. Liu, L. Zheng, and X. Huang, "Application of time reversal for TOA estimation in UWB ranging under dense multipath channel," in IEEE International Conference on Ultra-Wideband, Nov. 2013, pp. 61-65.

[28] F. Rachidi and M. Rubinstein, "Time reversal of electromagnetic fields and its application to lightning location," in International Symposium on Lightning Protection, Oct. 2013, pp. 378-383.

[29] W. M. G. Dyab, T. K. Sarkar, A. García-Lampérez, M. Salazar-Palma and M. A. Lagunas, "A Critical Look at the Principles of Electromagnetic Time Reversal and its Consequences," IEEE Antennas and Propagation Magazine, vol. 55, no. 5, pp. 28-62, Oct. 2013.

[30] A. G. Cepni, "Experimental Investigation of time-reversal techniques electromagnetic waves,” Ph. D. dissertation, Dep. Elect. Comp. Eng., Carnegie Mellon Univ., Pittsburgh, PA, 2005.

[31] S. He et al., "Norm Criteria in the Electromagnetic Time Reversal Technique for Fault Location in Transmission Lines," IEEE Transactions on Electromagnetic Compatibility, vol. 60, no. 5, pp. 1240-1248, Oct. 2018. 
[32] G. Lugrin, N. M. Mora, F. Rachidi, M. Rubinstein and G. Diendorfer, "On the Location of Lightning Discharges Using Time Reversal of Electromagnetic Fields," IEEE Transactions on Electromagnetic Compatibility, vol. 56, no. 1, pp. 149-158, Feb. 2014.

[33] W. Wu and Y. Xu, "Correlation analysis of visual verb' subcategorization based on Pearson's correlation coefficient," in International Conference on Machine Learning and Cybernetics, Jul. 2010, pp. 2042-2046.

[34] H. Zhu, X. You and S. Liu, "Multiple Ant Colony Optimization Based on Pearson Correlation Coefficient,” IEEE Access, vol. 7, pp. 61628-61638, 2019.

[35] J. W. McKown and R. L. Hamilton, "Ray tracing as a design tool for radio networks," IEEE Network, vol. 5, no. 6, pp. 27-30, Nov. 1991.

[36] T. Huschka, "Ray tracing models for indoor environments and their computational complexity," in 5th IEEE International Symposium on Personal, Indoor and Mobile Radio Communications, Wireless Networks - Catching the Mobile Future., Sept. 1994, pp. 486-490.

[37] C. Zhou and R. Jacksha, "Modeling and Measurement of Radio Propagation in Tunnel Environments," IEEE Antennas and Wireless Propagation Letters, vol. 16, pp. 1431-1434, 2017.

[38] A. Emslie, R. Lagace and P. Strong, "Theory of the propagation of UHF radio waves in coal mine tunnels," IEEE Transactions on Antennas and Propagation, vol. 23, no. 2, pp. 192-205, Mar. 1975.

[39] Shen Zhang, "The multipath propagation model of rectangular tunnel channel," in IEEE Region 10 Conference on Computers, Communications, Control and Power Engineering. TENCOM '02. Proceedings., Oct. 2002, pp. 1016-1019.

[40] Chenming Zhou and R. C. Qiu, "Spatial focusing of time-reversed UWB electromagnetic waves in a hallway environment," in Proceeding of the Thirty-Eighth Southeastern Symposium on System Theory, Mar. 2006, pp. 318-322.

[41] Y. P. Zhang and H. J. Hong, "Ray-optical modeling of simulcast radio propagation channels in tunnels," IEEE Transactions on Vehicular Technology, vol. 53, no. 6, pp. 1800-1808, Nov. 2004.

[42] P. R. Barnes and F. M. Tesche, "On the direct calculation of a transient plane wave reflected from a finitely conducting half space," IEEE Transactions on Electromagnetic Compatibility, vol. 33, no. 2, pp. 90-96, May.1991.

[43] R. C. Qiu, "A generalized time domain multipath channel and its application in ultra-wideband (UWB) wireless optimal receiver design-Part II: physics-based system analysis," IEEE Transactions on Wireless Communications, vol. 3, no. 6, pp. 2312-2324, Nov. 2004.

[44] D. Liu, S. Vasudevan, J. Krolik, G. Bal and L. Carin, "Electromagnetic Time-Reversal Source Localization in Changing Media: Experiment and Analysis," IEEE Transactions on Antennas and Propagation, vol. 55, no. 2, pp. 344-354, Feb. 2007.

[45] T. Wang, S. Qiu, L. Shi and Y. Li, "Broadband VHF Localization of Lightning Radiation Sources by EMTR,” IEEE Transactions on Electromagnetic Compatibility, vol. 59, no. 6, pp. 1949-1957, Dec. 2017. 


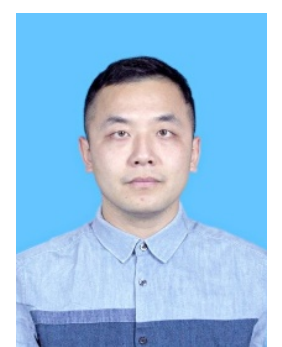

Qi Li was born in Weinan, Shannxi, China, in 1993. $\mathrm{He}$ received the B.Sc. degree in measurement techniques and instrumentation in 2015 and Ph.D. degree in electrical engineering in 2021 from Xi'an Jiaotong University, Xi'an, China. He is currently working at the Beijing Institute of Electronic System Engineering, Beijing, China. His research interests include the transient electromagnetic measurement and electromagnetic time reversal.

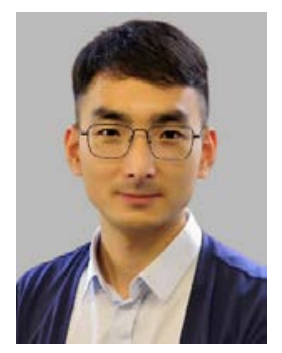

Zhaoyang Wang (M'17) received the M.Sc. degree in electrical engineering from Xi'an Jiaotong University, Xi'an, China, in 2015, and the Ph.D. degree in electrical engineering from the Swiss Federal Institute of Technology, Lausanne (EPFL), Switzerland, in 2020.

He is currently a Postdoctoral Research Fellow with the Electromagnetic Compatibility Laboratory and the Distributed Electrical Systems Laboratory of the Swiss Federal Institute of Technology, Lausanne (EPFL).

His research interests include electromagnetic transients, power system fault location and protection, electromagnetic time reversal, and electromagnetic transient disturbances localization.

He has been the recipient of the 2018 Richard B. Shultz Best Transaction Paper Award of IEEE Transactions on EMC and the URSI GASS 2021 Young Scientist Award.

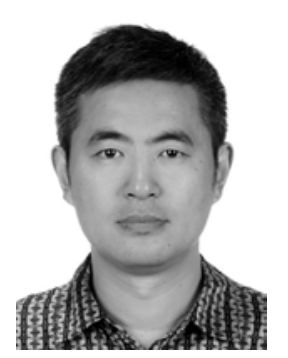

Yan-Zhao Xie (M'12-SM'19) was born in Xiayi, Henan, China, in 1973. He received the Ph.D. degree in electrical engineering from Tsinghua University, Beijing, China. He is currently a Full Professor in the School of Electrical Engineering, Xi'an Jiaotong University, Xi'an, China. His research interests include transient analysis of multi-conductor transmission lines, electromagnetic transients in power systems, electromagnetic time reversal and high power electromagnetics, etc.

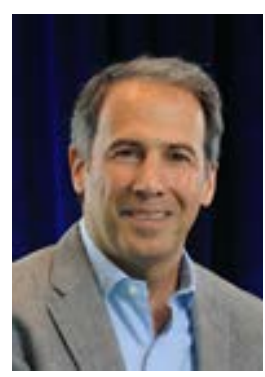

Farhad Rachidi (M'93-SM'02-F'10) received the M.S. and Ph.D. degrees in electrical engineering from the Swiss Federal Institute of Technology, Lausanne, Switzerland, in 1986 and 1991, respectively.

He was with the Power Systems Laboratory, Swiss Federal Institute of Technology, until 1996. In 1997, he joined the Lightning Research Laboratory, University of Toronto, Toronto, ON, Canada. From 1998 to 1999, he was with Montena EMC, Rossens, Switzerland. He is currently a Titular Professor and the Head of the EMC Laboratory, Swiss Federal Institute of Technology. He has authored or co-authored more than 170 scientific papers published in peer-reviewed journals and more than 350 papers presented at international conferences.

Dr. Rachidi is currently a Member of the Advisory Board of the IEEE TRANSACTIONS ON ELECTROMAGNETIC COMPATIBILITY and the President of the Swiss National Committee of the International Union of Radio Science. He was a recipient of numerous awards including the 2005 IEEE EMC Technical Achievement Award, the 2005 CIGRE Technical Committee Award, the 2006 Blondel Medal from the French Association of Electrical Engineering, Electronics, Information Technology and Communication, the 2016 Berger Award from the International Conference on Lightning Protection, the 2016 Best Paper Award of the IEEE Transactions on EMC, and the 2017 Motohisa Kanda Award for the most cited paper of the IEEE Transactions on EMC. In 2014, he was conferred the title of Honorary Professor of the Xi'an Jiaotong University, Xi'an, China. He served as the Vice-Chair of the European COST Action on the Physics of Lightning Flash and Its Effects from 2005 to 2009, the Chairman of the 2008 European Electromagnetics International Symposium, the President of the International Conference on Lightning Protection from 2008 to 2014, and the Editor-in-Chief for the Open Atmospheric Science Journal (2010-2012) and the IEEE TRANSACTIONS ON ELECTROMAGNETIC COMPATIBILITY from 2013 to 2015.

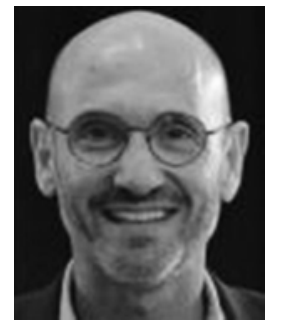

Marcos Rubinstein (M'84-SM'11-F'14) received the master's and Ph.D. degrees in electrical engineering from the University of Florida, Gainesville, FL, USA, in 1986 and 1991, respectively.

In 1992, he joined the Swiss Federal Institute of Technology, Lausanne, Switzerland, where he was involved in the fields of electromagnetic compatibility and lightning. In 1995, he was with Swisscom, where he worked in numerical electromagnetics and EMC. In 2001, he moved to the University of Applied Sciences of Western Switzerland HES-SO, Yverdon-les-Bains, where he is currently a Full Professor, Head of the Advanced Communication Technologies Group, and a Member of the IICT Institute Team. He is an author or co-author of more than 200 scientific publications in reviewed journals and international conferences. He is also the co-author of seven book chapters.

Dr. Rubinstein is the Chairman of the International Project on Electromagnetic Radiation from Lightning to Tall structures, served as the Editor-in-Chief of the Open Atmospheric Science Journal, and currently serves as an Associate Editor for the IEEE TRANSACTIONS ON ELECTROMAGNETIC COMPATIBILITY.

Prof. Rubinstein was the recipient of the Best Master's Thesis award from the University of Florida, and the IEEE achievement award. He is a co-recipient of the NASA's Recognition for Innovative Technological Work award. He is also the recipient of the ICLP Karl Berger Award. He is a Fellow of the SUMMA Foundation, a member of the Swiss Academy of Sciences and of the International Union of Radio Science. 